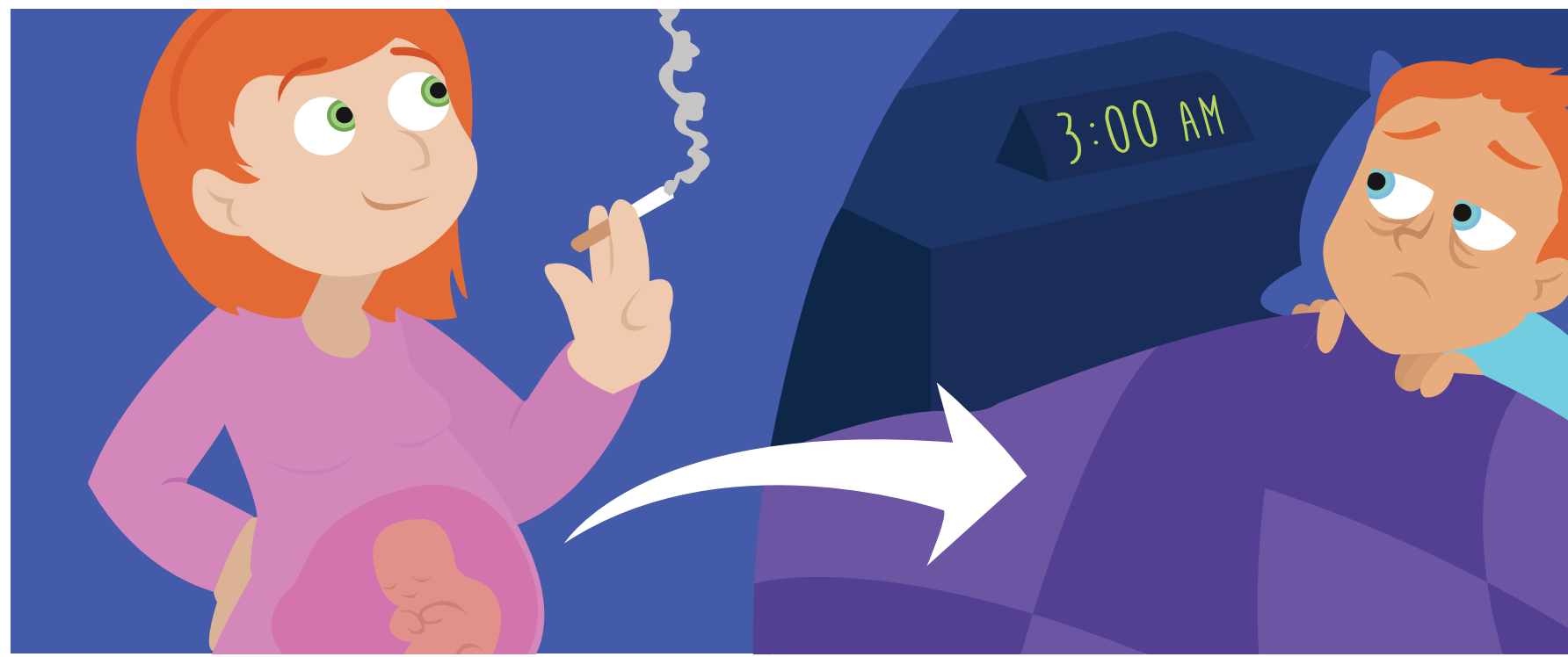

\title{
DO CIGARETTES HAVE LONG-LASTING EFFECTS ON CHILDREN'S SLEEP?
}

\section{Jeremy C. Borniger ${ }^{1 *}$, Reuben F. Don ${ }^{2}$, R. Thomas Boyd ${ }^{2}$, and Randy J. Nelson ${ }^{2}$}

${ }^{1}$ Department of Psychiatry and Behavioral Sciences, Stanford University, Stanford, CA, United States, ${ }^{2}$ Department of Neuroscience, The Ohio State University, Columbus, $\mathrm{OH}$, United States

\section{REVIEWED BY: \\ SECONDARY \\ $S C H O O L$ \\ "ALFREDO \\ FUSCO" \\ CASTELFORTE \\ AGE: 12-13}

Sleep is an important part of overall health and well-being. Without proper sleep, our thoughts get foggy, and we have trouble remembering things. On top of that, other parts of our bodies start to work less efficiently. For example, messed up sleep can cause changes in immune or metabolic systems that can promote disease or make certain diseases worse! Despite what we already know, not all the causes of sleep disruption are well understood. We all know now that smoking cigarettes is bad for your health, and a lot of evidence suggests that pregnant mothers who smoke cigarettes have children that have sleep problems. These problems may promote disease or interfere with life when the child grows up. Here, we will discuss three things: (1) How do we know what parts of the brain control sleep and how can we measure sleep in animals? (2) How do cigarettes alter the function of the brain and body? and (3) How can a smoking mother alter her child's sleep all the way into adulthood?

\section{WHAT PARTS OF THE BRAIN CONTROL SLEEP?}

The answer to this question was a mystery for a long time, and scientists are still putting the puzzle pieces together today. It was not until World War I 
ELECTROENCEPHALOGRAM (EEG)

A technique used to measure sleep in humans and animals. By measuring changes in voltage across the brain (difference in electrical charge between two points), scientists can gain an understanding of what the brain is doing.

\section{FIGURE 1}

Sleep can be measured using the electroencephalogram (EEG) to study an animal's brain. In this figure, you are looking at a side view of a mouse brain, with two electrodes implanted - they look like screws. The difference in charge (voltage) is measured between the two electrodes to produce the EEG signal you see in the table, associated with wake, non-REM, and REM sleep. In the brain cartoon, you can see that many different neurotransmitters (colored circles) are involved in controlling sleep and wakefulness, including acetylcholine. Cells that make these neurotransmitters are located in different parts of the brain, but the neurotransmitters travel to distant brain locations to bind their receptors.
(1914-1918) that we started to understand where in the brain sleep is controlled. A doctor named Constantin von Economo noticed that some of his patients with damage to a certain part of the brain (called the posterior hypothalamus) would sleep all day, and that others with damage to another part of the brain (called the anterior hypothalamus) would have a very hard time falling sleep. The cause of this brain damage is still unknown! Based on these observations, Dr. Economo predicted that cells in the anterior hypothalamus promote sleep and those in the posterior hypothalamus promote wakefulness. In more recent years, the specific cells in these regions controlling different aspects of sleep have been identified and manipulated.

After Dr. Economo's observations, others started to use electrodes (a conductive piece of metal used to contact a non-metallic part of a circuit) to monitor and alter brain activity in living animals. Because the brain communicates using small electrical signals, measuring changes in voltage (which is a measure of the difference in electrical charge between two points) throughout the brain can give us an understanding of what the brain is doing. One way to look at brain activity using voltage measurements is through something called the electroencephalogram (EEG) (Figure 1). As few as two electrodes are placed onto the scalp or the surface of the brain, and the difference in charge is measured between the two points. In the late 1940s, Drs. Giuseppe Moruzzi and Horace Magoun used this technique to discover a group of cells (called the reticular activating system) in the lower part of the brain that control wakefulness. They stimulated this group of cells in the brain of a cat, while measuring EEG signals, and discovered that waking up from sleep was reflected in the EEG, where

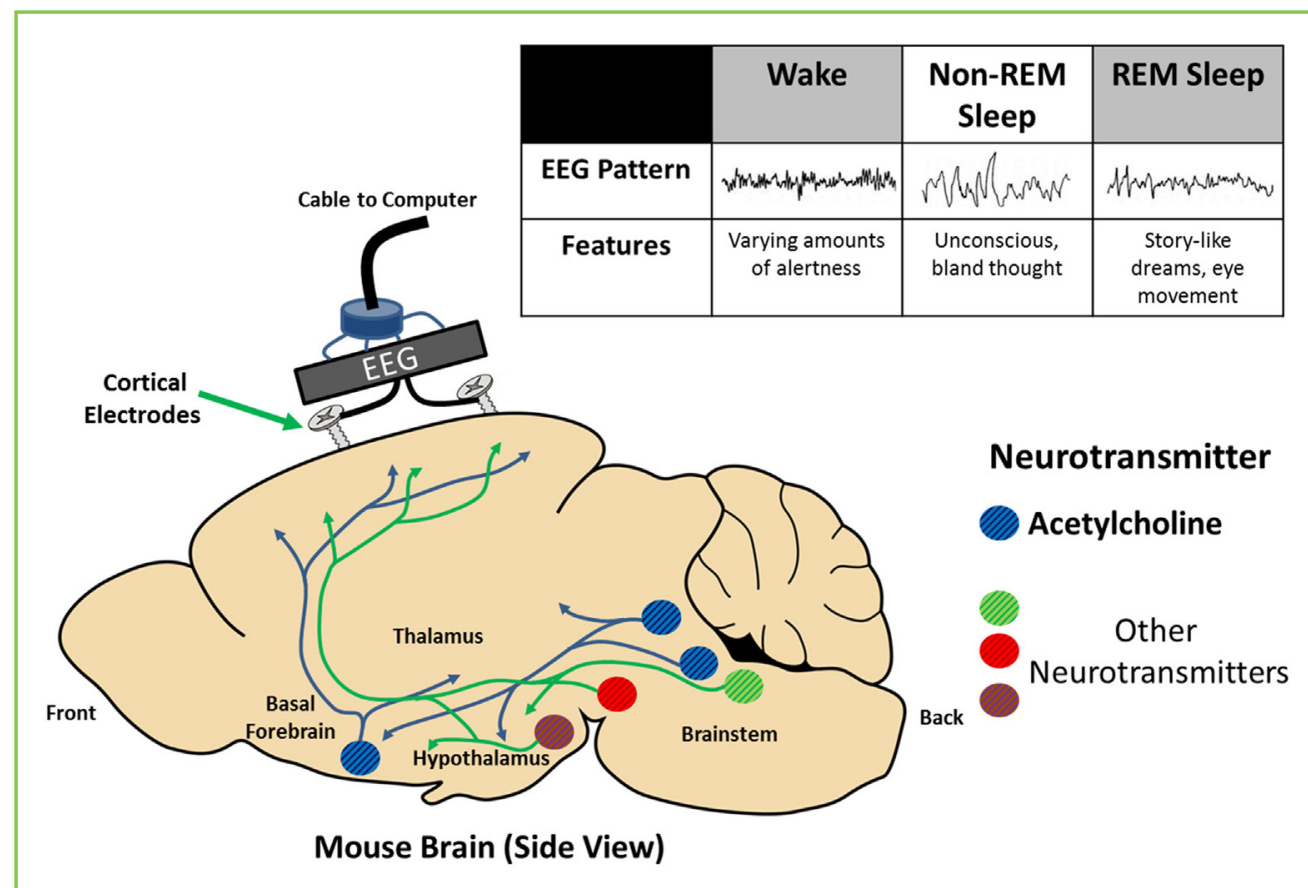

FIGURE 1 


\section{NEUROTRANS- \\ MITTER}

A neurochemical message released from neurons (brain cells) to communicate with other cells, thereby changing their behavior. Some well-known neurotransmitters include acetylcholine, dopamine, serotonin, and norepinephrine.

\section{ACETYLCHOLINE}

An important neurotransmitter (neurochemical message) involved in sleep and wakefulness, as well as in immune function.

\section{FIGURE 2}

Nicotine changes how immune and brain cells (neurons) behave, through binding to nicotinic acetylcholine receptors. In this figure, you can see that nicotine (red shape) or acetylcholine (green shape) binding to immune cells can reduce their activity (symbolized by a down arrow). Alternatively, nicotine or acetylcholine binding to neurons can cause them to become activated (up arrow) and to release neurotransmitters. These neurotransmitters can affect lots of things, including immune function and sleep. voltage measurements went from large to small. This means that we can tell whether an animal (or human) is sleeping or waking just by looking at the changes in voltage over the entire brain! Even more interestingly, we can determine whether someone is in deep or light sleep from these EEG measurements, making the EEG very useful for examining sleep in both animals and people.

Building on these findings, scientists have begun to pinpoint specific molecules (called neurotransmitters) that control sleep and wakefulness by acting on cells in the hypothalamus and reticular activating system. Neurotransmitters are molecules produced by brain cells (neurons) that send chemical messages to other cells to alter their function. One of the most common neurotransmitters in the brain and body is called acetylcholine [1]. Acetylcholine is produced by many different types of neurons, including those that control sleep and wakefulness. Acetylcholine acts on cells by binding to a molecule called an acetylcholine receptor, similar to a key fitting into a lock (Figure 2). Acetylcholine produced by specific cells in the brainstem is critical for a type of sleep called "rapid eye movement" (REM) sleep. During this type of sleep, your eyes move back and forth and the EEG pattern looks like it does when you are awake (even though you are in deep sleep)! This type of sleep is frequently associated with dreaming, because if people are awakened immediately after REM sleep episodes, they say that they were dreaming. If scientists inject a drug that mimics the actions of acetylcholine (called carbachol) into the brainstem, then they can cause REM sleep in animals. Acetylcholine produced in another part of the brain (called the basal forebrain) is a strong regulator of sleep as well, and destruction of cells in this brain region can severely affect sleep. This is all interesting, but how do cigarettes fit into this story?

\section{HOW DO CIGARETTES ALTER BRAIN AND BODY FUNCTION?}

Cigarette smoke is made up of thousands of different components with many different effects. Nicotine is the primary component in cigarettes that is

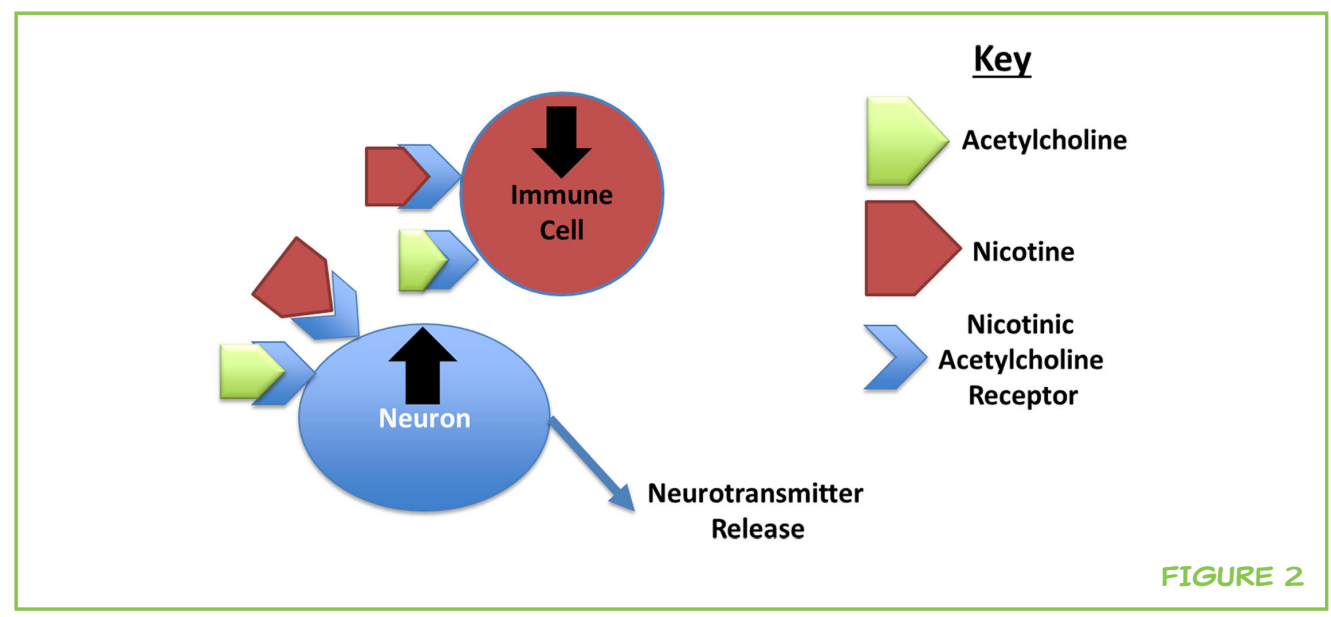




\section{RECEPTOR}

A site on a cell where specific molecules (like acetylcholine or nicotine) bind. The receptor is specific, the way a key only fits in a certain lock. Molecules binding the receptor cause the cell to change its behavior. Nicotinic acetylcholine receptors are subtypes of receptors that bind both acetylcholine and nicotine.

\section{REM SLEEP}

Rapid eye movement sleep is an active state of sleep characterized by a wake-like EEG pattern and is widely associated with dreaming.

Acetylcholine is important for the generation of REM sleep in mice and humans.

\section{NICOTINE}

A component of cigarettes that is primarily responsible for their addicting and stimulating properties. Nicotine can mimic the actions of acetylcholine on many cells in the body, by binding nicotinic acetylcholine receptors.

\section{LIPOPOLYSAC-} CHARIDE (LPS)

A (harmless) piece of bacteria that can cause a severe immune reaction if given to humans or animals. LPS allows scientists to investigate how the immune system contributes to changes in behaviors associated with sickness, such as sleepiness, depression, and reductions in social interactions. responsible for their habit-forming or addictive properties. It mimics the action of acetylcholine at specific acetylcholine receptors. Therefore, nicotine can activate many of the same cells that acetylcholine does. Nicotine is a natural insecticide produced by the nightshade family of plants (of which the tobacco plant is a member), to prevent bugs from eating them. Nicotine acts through binding nicotinic acetylcholine receptors on the surface of different cells in the body and brain. For bugs, the effect of nicotine is poisonous, preventing them from eating the plant. However, in humans, the effect of nicotine can be stimulating, and therefore addicting. Because these receptors are on many different cells throughout the body, nicotine can have wide-ranging effects on many different body systems. For example, nicotine can alter the function of many different cells in the hypothalamus and other parts of the brain that control sleep and wakefulness through acting on nicotinic acetylcholine receptors. If too much stimulation occurs, this can alter how the cells behave normally, and therefore how they act even in the absence of nicotine. Nicotine does not just alter brain function, but can also change how cells in other parts of the body work. For example, many cells of the immune system have nicotinic receptors on their surface, making them sensitive to the effects of nicotine. In general, nicotine suppresses immune function through acting on these receptors. This may be a reason why people who smoke cigarettes get sick more often than those that do not smoke.

Mothers who smoke cigarettes during pregnancy have children who are at an increased risk for many different conditions, including sleep problems. However, it is unknown if exposure to nicotine early in life (or exposure to other compounds in cigarettes) actually causes development of sleep problems in children born to smoking mothers. Therefore, an important question to answer is whether early-life nicotine alters sleep directly, and how this happens. With this knowledge, we can gain a better understanding of sleep disruption and how to prevent the bad effects associated with it.

\section{OF MICE AND MEN}

Using pregnant women to test the role of nicotine in the development of sleep problems is not allowed, because we know that nicotine can cause adverse effects and hurt the baby. Because of these problems, scientists frequently rely on using laboratory mice to investigate biological questions. In some ways, mice are very similar to humans and they respond to many drugs and substances the same way humans do. This makes laboratory mice a great model organism to use to test whether nicotine use during pregnancy directly alters the sleep of the offspring mice, even into adulthood.

To do this, researchers gave pregnant mice nicotine dissolved in sweetened water (we called this the treatment) or just sweetened water with no nicotine 


\section{FIGURE 3}

Mice exposed to nicotine during early-life spent less time awake A. In panel A, each dot represents the percent of time one mouse spent awake during the day and night (shaded area). The spread of these dots show the distribution of the data. Control mice are represented by black dots and nicotine-exposed mice by red dots. The bars and lines behind the dots represent the mean and spread of the data to summarize what the dots show. Notice the distribution of the data and the large difference in wakefulness between the day and night (mice are nocturnal, so they spend most of the night awake). B. Nicotine-exposed mice entered less deep sleep after receiving lipopolysaccharide (LPS) than did mice that did not receive nicotine in early life. LPS was given at the time indicated by the arrow in panel B. Depth of sleep was evaluated based on the prevalence of low frequency components in the electroencephalogram signal. The shaded area in panel $\mathbf{B}$ indicates time when lights were off. These data indicate that nicotine exposure in early life made mice sleep more and have a blunted sleep response to an immune challenge when they grew up. (we called this the control) during the whole pregnancy [2]. They needed to sweeten the water because nicotine tastes bad! Because mice grow at a different rate than humans, the researchers continued nicotine (or control) treatment until the baby mice were 3 weeks old. Then, they stopped treatment and allowed the mice to drink normal water for the rest of the experiment. This means that the baby mice were only exposed to nicotine during a very early stage in their development (similar to human babies with a smoking mom). Once the mice grew up (about 10 weeks old, equivalent to 13- to 15-year-old humans), the scientists used EEG monitors to examine the sleep patterns of the mice. After recording sleep for a few days, the researchers challenged the mice's sleep system by depriving them of sleep for $5 \mathrm{~h}$ (similar to staying up really late), and then looking at how the mice recovered from this challenge. They observed that, in general, mice that had been exposed to nicotine slept more than normal. These mice also showed increased sleep in response to sleep deprivation compared with mice that were not exposed to nicotine in early life.

As a final test, the researchers gave mice a challenge to their immune systems using a molecule called lipopolysaccharide (LPS). LPS is a (harmless) component of certain bacteria, so if your immune system encounters it, it will respond as if you have a bacterial infection even though there are no actual bacteria present. This immune response causes you to feel sick and tired, requiring a lot of sleep to recover. Because the researchers knew that nicotine can affect the immune system, they wanted to see whether early-life nicotine exposure would interfere with the normal sleep response to LPS. Mice that had received nicotine during early life showed less deep sleep after LPS compared with mice that were not exposed to nicotine (Figure 3).

\section{WHAT CONCLUSIONS CAN BE MADE?}

What do you think could explain these findings? The researchers believe that the changes they saw in mouse sleep in response to early-life nicotine could be due to long-lasting changes in the function of nicotinic acetylcholine receptors

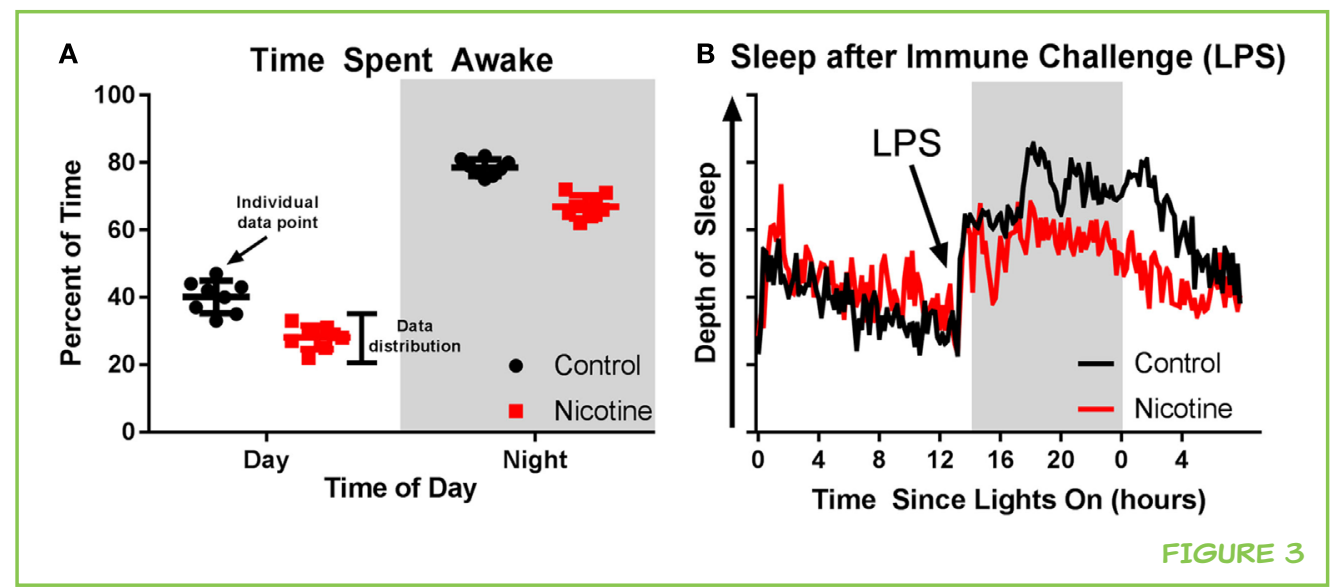


on the cells that control sleep and waking in the brain. Activation of nicotinic acetylcholine receptors by nicotine at the wrong time could lead to cell death or disruption of the normal signaling that occurs during development. What could be a good follow up experiment to test whether this was the case? It is likely that, in response to LPS, nicotine-exposed mice did not sleep as much because their immune systems were suppressed by the early-life nicotine treatment. How could this be happening?

In addition to possible effects on the nicotinic acetylcholine receptors, there are some other ideas as to why sleep may be changed all the way into adulthood in response to nicotine. The nicotine was given to pregnant mice, which then gave birth to and took care of the baby mice during the first 3 weeks of their lives. Perhaps nicotine interfered with normal mothering behavior, which could have long-lasting effects on the baby mice as they grew up. Finally, there are some well-known effects of nicotine on breathing and on brain cells involved in the control of breathing. Perhaps the effects on sleep are just a side effect of nicotine's effects on breathing. What could be some tests that would let the scientists know whether mother behavior or breathing was affected by nicotine treatment? For example, scientists could expose pregnant mice to nicotine as was done in the experiments discussed earlier, and then investigate how they care for their offspring. If they hypothesized that the mother's behavior was important for the offspring mouse development, then they could let a mother mouse that was not exposed to nicotine raise mice that were, therefore controlling for the mother's behavior while keeping the nicotine variable constant. Together, these experiments suggest that early-life exposure to nicotine can have long-lasting effects on sleep into adulthood. Because sleep is such an important part of health, doctors may want to further guard against nicotine use during pregnancy, to prevent the development of sleep problems in children and adults.

\section{ORIGINAL SOURCE ARTICLE}

Borniger, J. C., Don, R. F., Zhang, N., Boyd, R. T., and Nelson, R. J. 2017. Enduring effects of perinatal nicotine exposure on murine sleep in adulthood. Am. J. Physiol. 313, R280-89. doi:10.1152/ajpregu.00156.2017

\section{REFERENCES}

1. Scammell, T. E., Arrigoni, E., and Lipton, J. O. 2017. Neural circuitry of wakefulness and sleep. Neuron 93, 747-65. doi:10.1016/j.neuron.2017.01.014

2. Borniger, J. C., Don, R. F., Zhang, N., Boyd, R. T., and Nelson, R. J. 2017. Enduring effects of perinatal nicotine exposure on murine sleep in adulthood. Am. J. Physiol. 313, R280-89. doi:10.1152/ajpregu.00156.2017 
SUBMITTED: 23 November 2017; ACCEPTED: 09 February 2018;

PUBLISHED ONLINE: 13 March 2018.

EDITED BY: Valeria Costantino, University of Naples Federico II, Italy

CITATION: Borniger JC, Don RF, Boyd RT and Nelson RJ (2018) Do Cigarettes Have Long-lasting Effects on Children's Sleep? Front. Young Minds 6:7. doi:10.3389/ frym.2018.00007

CONFLICT OF INTEREST STATEMENT: The authors declare that the research was conducted in the absence of any commercial or financial relationships that could be construed as a potential conflict of interest.

COPYRIGHT (C) 2018 Borniger, Don, Boyd and Nelson. This is an open-access article distributed under the terms of the Creative Commons Attribution License (CC BY). The use, distribution or reproduction in other forums is permitted, provided the original author(s) and the copyright owner are credited and that the original publication in this journal is cited, in accordance with accepted academic practice. No use, distribution or reproduction is permitted which does not comply with these terms.

\section{REVIEWED BY}

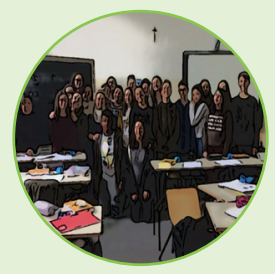

\section{SECONDARY SCHOOL "ALFREDO FUSCO" CASTELFORTE, AGE: 12-13}

The reviewers are a group of young science enthusiasts of the class 3B, ages ranging from 12 to 13 years, of a Secondary School "A. FUSCO" located in Castelforte (LT), Italy. From this practice, we have learned: what are the long-lasting effects of the nicotine exposure in early life on children's or adulthood sleep. We also enjoyed learning about the methods used in the scientific research, asking questions, and making suggestions to help the scientists to make their work easier and understandable to everyone. The review project was carried out with help of our science educators Barbara lovine, Maria Amato, as well the science mentor M. Assunta Bevilacqua.

\section{AUTHORS}

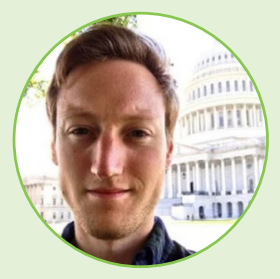

\section{JEREMY C. BORNIGER}

Jeremy C. Borniger was born in Washington, DC, USA and attended Indiana UniversityBloomington for his undergraduate degree. He then completed his $\mathrm{PhD}$ in neuroscience in Randy J. Nelson's lab at The Ohio State University. His primary focus was on how tumors contribute to changes in sleep and wakefulness as well as metabolism. Jeremy C. Borniger is now a postdoctoral fellow in Luis de Lecea's lab at Stanford University. In his spare time, Jeremy C. Borniger likes playing the piano, scuba diving, running, and reading. *jcbornig@stanford.edu 


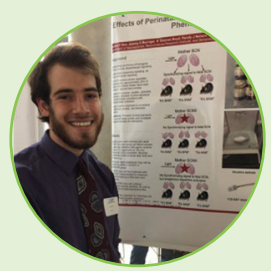

\section{REUBEN F. DON}

As a neuroscience major at The Ohio State University, Reuben focused on how nicotine use during pregnancy affected offspring sleep in adulthood. He also organized twice-monthly discussion groups to talk about recent publications in the field of pharmacology. He will soon start a position as a research associate at the National Institutes of Drug Abuse, studying the mechanisms of drug addiction. In his free time, he enjoys hiking, art, rock climbing, and live music.

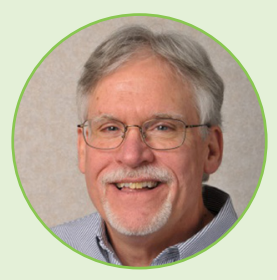

\section{R. THOMAS BOYD}

Dr. R. Thomas Boyd graduated from the University of Illinois at Urbana-Champaign in 1977 with a BS. He received a PhD in from the University of Texas at Austin in 1986. After working as a postdoctoral researcher at the University of California, San Diego and the Salk Institute for 4 years, he moved to Ohio State in 1990. He is an associate professor in the Department of Neuroscience. Research activities in his laboratory have emphasized biological analysis of neuronal nicotinic acetylcholine receptors (nAChRs), including the cloning of neuronal nAChRs from zebrafish.

\section{RANDY J. NELSON}

Randy J. Nelson is a Distinguished University Professor and Chair of the Department of Neuroscience at The Ohio State University Wexner Medical Center. He holds the Brumbaugh Chair in Brain Research and Teaching and co-directs the Neuroscience Research Institute. Nelson earned his AB, a PhD in Psychology, as well as a second PhD in Endocrinology from UC Berkeley, the first to earn two PhDs simultaneously in the US. He then completed work at the University of Texas, Austin. Randy J. Nelson was on the faculty at Johns Hopkins from 1986 to 2000 and then moved to Ohio State. He has published $>400$ scientific articles and a dozen books describing studies in biological rhythms, sleep, and behavioral neuroendocrinology. 